\title{
Improved Diastolic Dysfunction is Associated with Higher Forward Flow and Better Prognosis in Chronic Heart Failure
}

Frank L Dini

AOUP: Azienda Ospedaliero Universitaria Pisana

Piercarlo Ballo

USL Tuscany centre: Azienda USL Toscana centro

Nicola Riccardo Pugliese ( $\nabla$ n.r.pugliese88@gmail.com )

University of Pisa https://orcid.org/0000-0002-5473-227X

Ibadete Bytyçi

Umea University Faculty of Medicine: Umea Universitet Medicinska fakulteten

Andreina D'Agostino

AOUP: Azienda Ospedaliero Universitaria Pisana

Gani Bajraktari

Umea University Faculty of Medicine: Umea Universitet Medicinska fakulteten

Roberto Pedrinelli

AOUP: Azienda Ospedaliero Universitaria Pisana

Michael Henein

Umea University Faculty of Medicine: Umea Universitet Medicinska fakulteten

\section{Research Article}

Keywords: heart failure, echocardiography, prognosis.

Posted Date: August 6th, 2021

DOl: https://doi.org/10.21203/rs.3.rs-769194/v1

License: (c) (i) This work is licensed under a Creative Commons Attribution 4.0 International License. Read Full License

Version of Record: A version of this preprint was published at The International Journal of Cardiovascular Imaging on November 2nd, 2021. See the published version at https://doi.org/10.1007/s10554-02102457-z. 


\section{Abstract}

Aim. In patients with chronic heart failure (HF), the benefit of repeating the assessment of left ventricle (LV) systolic and diastolic function over time remains uncertain. We assessed the prognostic value of repeated echocardiographic assessment of LV filling pressure (LVFP) and its interaction with cardiac index $(\mathrm{Cl})$ in ambulatory patients with chronic $\mathrm{HF}$ and reduced ejection fraction (HFrEF)

Methods and results. We enrolled 367 patients (age $68 \pm 11$ years; $22 \%$ female) with chronic HFrEF. Patients underwent a clinical and echocardiographic examination at baseline and were re-evaluated after $6 \pm 3$ months. The 2016 recommendations were used to estimate normal or increased LVFP. Cl was evaluated as the product of LV outflow tract area and velocity-time integral multiplied by heart rate and divided by body surface area. After the second examination, patients were followed for a median of 30 months. The study endpoint included all-cause death and hospitalization for worsening HF. Patients who normalized LVFP or showed persistently normal LVFP at the follow-up examination had a significantly lower mortality rate than those with worsening or persistently raised LVFP. After further stratification by $\mathrm{Cl}$, patients with elevated LVFP and $\mathrm{Cl}<2.0 \mathrm{~L} / \mathrm{min} / \mathrm{m}^{2}$ had a further worse outcome than those with elevated LVFP and $\mathrm{Cl} \geq 2.0 \mathrm{~L} / \mathrm{min} / \mathrm{m}^{2}$. Multivariate survival analysis confirmed an independent prognostic impact of changes in LVFP, incremental to that of established clinical, laboratory and echocardiographic predictors.

Conclusions. Repeated evaluation based on a full diastolic function assessment of LVFP and $\mathrm{Cl}$ significantly improved risk stratification of stable HFrEF outpatients compared to baseline evaluation.

\section{Background}

Management of heart failure (HF), improvement of survival and prevention of hospitalizations largely depend on LV structural and/or functional cardiac abnormalities, resulting in a reduced cardiac output (CO) and/or elevated intracardiac pressures at rest or during stress. Currently, Doppler echocardiography can provide reliable and reproducible LV filling pressure measures (LVFP) and forward flow, i.e., stroke volume and $\mathrm{CO}[1]$.

Several echocardiographic studies have shown that markers of elevated LVFP have been associated with an adverse prognosis[2], but their reversal toward normality with treatment favourably impact clinical outcome[3-5]. The EAEVI Euro-Filling Study has demonstrated that the 2016 ASE/EACVI algorithms are reliable and clinically useful for non-invasively estimating LVFP[6]. Still, the impact of normalization and persistently elevated LVFP at follow-up on prognosis in patients with chronic HF remains unknown. Since diastolic dysfunction (DD) plays an important role in determining $\mathrm{CO}[7,8]$ and because a decreased forward flow is negatively associated with prognosis $[9,10]$, the present study was designed to ascertain: 1) the prognosis of ambulatory HF patients with persistent or worsening DD as assessed by the 2016 recommendations, 2 ) whether the recovery of DD during follow-up was associated with improved 
patients' clinical outcome; 3 ) the prognostic impact of reduced forward flow in patients with persistent or worsening DD at follow-up.

\section{Patients And Methods}

Study patients. This prospective study included consecutive patients with chronic HF referred to our outpatient clinics in Pisa for clinical evaluation and follow-up from 2010 to 2019 . The inclusion criteria comprised LV EF $\leq 50 \%$ and $L V$ end-diastolic volume (EDV) index $>75 \mathrm{ml} / \mathrm{m}^{2}$.

Exclusion criteria were: recent myocardial infarction or unstable angina ( $<3$ months), coronary artery bypass graft or percutaneous coronary angioplasty ( $<3$ months), more than mild aortic or organic mitral valve disease, cardiac valve lesions requiring surgical and/or percutaneous repair, planned cardiac resynchronization therapy, hypertrophic cardiomyopathy, amyloidosis, active myocarditis, prosthetic valve dysfunction, and inadequate image quality. Patients with atrial fibrillation were not excluded from the study. The study was approved by the local institutional review board. All patients gave written informed consent with their approval to participate in the study. The study was conducted in accordance with the institutional policies, national legal requirements, and the revised Helsinki declaration.

Clinical evaluation. The patient's functional status was determined according to the classification of the New York Heart Association (NYHA). Blood was drawn for natriuretic peptides (NP), B-type natriuretic peptide (BNP) and amino-terminal proB-type natriuretic peptide (NT-proBNP) assays, and measure of renal function. Blood pressure, heart rate and rhythm were recorded. The estimated glomerular filtration rate was calculated from the simplified formula derived from the Modification of Diet in Renal Disease (MDRD) study.

Echocardiographic examination. Transthoracic two-dimensional and Doppler echocardiographic examination (including tissue Doppler) was carried out with an $\mathrm{iE} 33 \mathrm{X5}$-matrix and an EPIQ 7 Ultrasound instrument (Philips, Andover, Massachusetts) equipped with a 3.5-MHz transducer. The following standard echocardiographic variables were obtained: LV volumes and EF and left atrial volume index (LAVi), were assessed from apical two- and four-chamber views using the biplane Simpson's method. From mitral velocity tracings, peak early $(E)$ and late $(A)$ transmitral flow velocities, their ratio $E / A$, and $E$ wave deceleration time (EDT) were measured from spectral Doppler recordings of LV filling. Doppler tissue imaging longitudinal velocities were recorded with the sample volume placed at the junction between the septal and lateral LV wall and the mitral annulus in the 4-chamber view. A ratio of E/averaged myocardial early velocity of the lateral and septal sites (averaged E/e') was calculated. Tricuspid annulus plane systolic excursion (TAPSE) was measured from M-mode recordings. Retrograde blood flow of tricuspid regurgitation was recorded using continuous-wave Doppler for measuring peak tricuspid regurgitation velocity (TRV). The peak velocity was assigned as the average of five tricuspid regurgitation envelopes with the greatest maximal velocities and spectral density. The ASE-EACVI 2016 recommendations were used to estimate normal or increased LVFP[11] The LV outflow tract (LVOT) anteroposterior diameter was measured in the parasternal long-axis view, and the LVOT area was 
estimated as $\pi(d / 2)^{2}\left(\mathrm{~cm}^{2}\right)$. LV stroke volume was calculated as the product of the LVOT area and the velocity-time integral of the forward flow. LVOT velocity-time integral was measured by tracing the outer edge of the densest (or brightest) portion of the spectral tracing with the pulsed wave Doppler sample volume positioned in the middle of the LVOT below the aortic cusps from the apical 5-chamber or longaxis view, and $\mathrm{CO}$ was calculated as stroke volume multiplied by heart rate[12]. Cardiac index (Cl) was estimated by dividing $\mathrm{CO}$ by body surface area. For each Doppler-based measurement, estimates were obtained from 3 cardiac cycles in sinus rhythm or 5 in patients with atrial fibrillation. Mitral regurgitation severity was graded using the vena contracta method or by measuring the effective regurgitant orifice area into mild, moderate and severe.

Echocardiographic hemodynamic classification. Study patients were classified according to the presence or absence of DD in the light of ASE-EACVI 2016 recommendations. Patients with elevated LVFP were considered those with $E / A \geq 2.0$ or exhibiting $>50 \%$ of the following criteria: an average (of septal and lateral) $\mathrm{E} / \mathrm{e}^{\prime}>14$, a septal $\mathrm{e}^{\prime}<7 \mathrm{~cm} / \mathrm{c}$ or lateral $<10 \mathrm{~cm} / \mathrm{s}$, a TRV $>2.8 \mathrm{~m} / \mathrm{s}$ and a LAVi $>34 \mathrm{ml} / \mathrm{m}^{2}$. LVFP was considered normal if $<50 \%$ of previous criteria were present or when $E / A \leq 0.8$ or $E \leq 50 \mathrm{~cm} / \mathrm{s}$. When ASEEACVI 2016 recommendations could not determine DD, identification of elevated LVFP relied on an algorithm based on non-invasively measured pulmonary diastolic pressure[13]. Patients were defined as having normal forward flow if they had $\mathrm{Cl} \geq 2.0 \mathrm{l} / \mathrm{min} / \mathrm{m}^{2}$, whilst a reduced forward flow was characterized by $\mathrm{Cl}<2.0 \mathrm{l} / \mathrm{min} / \mathrm{m}^{2}$.[9]

Follow up. Patients were evaluated at baseline (index echocardiogram) and underwent clinical evaluation, BNP or NT-proBNP assessment and repeated echocardiographic examinations $6 \pm 3$ months afterwards. At follow-up, patients were classified into four groups based on changes in LV diastolic function: 1) persistently normal, if the diastolic function was normal on both baseline and follow-up exams; 2 ) reversible, if DD was present only at baseline; 3 ) worsening, if DD was present only at follow-up; 4) persistently abnormal, if the diastolic function was abnormal on both baseline and follow-up examinations. Abnormal natriuretic peptide concentration at follow-up was defined as BNP $\geq 150 \mathrm{pg}$ / $\mathrm{mL}$ or NT-proBNP $\geq 450 \mathrm{pg} / \mathrm{mL}$, along with failure to demonstrate $\geq 30 \%$ reduction from baseline[14].

Study endpoints. The endpoint was a composite of all-cause mortality and HF hospitalization. Survival data were obtained through follow-up visits of patients or, in the case of missed visits, through telephone contacts. Follow-up data were obtained by reviewing the patient's hospital records; death certificates were obtained in case of need. Survival analyses were performed considering the day of the follow-up examination as the starting day. For patients without events, the date of the last contact was considered the end of follow-up for survival analysis.

Statistical analysis. Data for continuous variables were presented as mean \pm SD or as median with interquartile range in case of non-normal distribution. Categorical variables were presented as number and percentage. Differences in continuous variables among groups were analyzed by the Student's $t$-test or ANOVA, as appropriate. For non-parametric variables, the Mann-Whitney $U$ test and the Kruskal-Wallis test were used. Categorical variables were compared by the Chi-square test or Fisher exact probability test 
when indicated. Two-way repeated-measures ANOVA was used to assess the effect of LV changes in LV diastolic function on $\mathrm{Cl}$. Kaplan-Meier curves were plotted to assess event-free survival probability across groups. Multivariate Cox proportional hazards regression was used to estimate the prognostic value of changes in LV diastolic function. Model discrimination was explored by receiver operating characteristic (ROC) analysis using the linear predictor score obtained from the proportional hazards model. The likelihood ratio test was considered to compare the performance among nested models and assess the incremental value of LV diastolic function changes over established predictors of outcome. Data were analyzed using the IBM SPSS Statistics software, v. 24. Differences were considered statistically significant for $\mathrm{P}<0.05$.

\section{Results}

The study population included 367 patients (mean age $68 \pm 11$ years; $22 \%$ female). Table 1 shows the main characteristic of the study patients. $44 \%$ of patients had ischemic LV dysfunction, $31 \%$ were in NYHA I, $49 \%$ in class NYHA II, and $20 \%$ in class NYHA III. According to the study design, all patients had LV systolic dysfunction (mean LV EF $33 \pm 7$, range $11-50 \%$ ); $31 \%$ had severe LV dysfunction, defined as $\mathrm{EF}<30 \%$. LV DD, assessed separately at baseline or the follow-up examination (Fig. 1), was an independent predictor of outcome, also after stratification by $\mathrm{Cl}$ (Supplemental Figs. 1-4). 
Table 1

Characteristics of the study patients

\begin{tabular}{|c|c|}
\hline Variable & Overall population $(n=357)$ \\
\hline Age (years) & $68.2 \pm 11.4$ \\
\hline Systolic blood pressure $(\mathrm{mmHg})$ & $124 \pm 17$ \\
\hline Diastolic blood pressure $(\mathrm{mmHg})$ & $74 \pm 9$ \\
\hline Mean blood pressure $(\mathrm{mmHg})$ & $91 \pm 11$ \\
\hline Creatinine (mg/dL) & $1.2 \pm 0.5$ \\
\hline $\operatorname{MDRD}\left(\mathrm{ml} / \mathrm{min} / 1.73 \mathrm{~m}^{2}\right)$ & $69.7 \pm 24.6$ \\
\hline Weight (kg) & $80 \pm 17$ \\
\hline $\operatorname{BSA}\left(\mathrm{m}^{2}\right)$ & $1.9 \pm 0.2$ \\
\hline $\mathrm{BMI}\left(\mathrm{kg} / \mathrm{m}^{2}\right)$ & $27.2 \pm 5$ \\
\hline \multicolumn{2}{|l|}{ Echocardiography at baseline } \\
\hline Heart rate (bpm) & $71 \pm 13$ \\
\hline $\mathrm{E}(\mathrm{cm} / \mathrm{s})$ & $75.5 \pm 26$ \\
\hline $\mathrm{A}(\mathrm{cm} / \mathrm{s})$ & $77.8 \pm 27$ \\
\hline $\mathrm{E} / \mathrm{A}$ & $1.1 \pm 1$ \\
\hline Deceleration time (ms) & $181 \pm 65$ \\
\hline$E / e^{\prime}$ & $14 \pm 6$ \\
\hline $\mathrm{LAVi}\left(\mathrm{mL} / \mathrm{m}^{2}\right)$ & $42.5 \pm 17$ \\
\hline sPAP $(\mathrm{mmHg})$ & $37 \pm 11$ \\
\hline $\operatorname{EDVi}\left(\mathrm{mL} / \mathrm{m}^{2}\right)$ & $99.9 \pm 28$ \\
\hline ESVi $\left(\mathrm{mL} / \mathrm{m}^{2}\right)$ & $68 \pm 25$ \\
\hline LVEF (\%) & $33 \pm 7$ \\
\hline Stroke volume (mL) & $65 \pm 17$ \\
\hline Cardiac index $\left(\mathrm{L} / \mathrm{min} / \mathrm{m}^{2}\right)$ & $2.3 \pm 0.5$ \\
\hline TAPSE (mm) & $19 \pm 4$ \\
\hline \multicolumn{2}{|l|}{ Echocardiography at follow-up } \\
\hline Heart rate (bpm) & $72 \pm 13$ \\
\hline
\end{tabular}




\begin{tabular}{|ll|}
\hline Variable & Overall population $(\mathbf{n}=\mathbf{3 5 7})$ \\
\hline $\mathrm{E}(\mathrm{cm} / \mathrm{s})$ & $76 \pm 26$ \\
\hline ( $(\mathrm{cm} / \mathrm{s})$ & $77 \pm 27$ \\
\hline E/A & $1.2 \pm 1$ \\
\hline Deceleration time $(\mathrm{ms})$ & $183 \pm 67$ \\
\hline E/e' & $14 \pm 6$ \\
\hline LAVi $\left(\mathrm{mL} / \mathrm{m}^{2}\right)$ & $43.5 \pm 18$ \\
\hline sPAP $(\mathrm{mmHg})$ & $36 \pm 10$ \\
\hline EDVi $\left(\mathrm{mL} / \mathrm{m}^{2}\right)$ & $95 \pm 28$ \\
\hline ESVi $\left(\mathrm{mL} / \mathrm{m}^{2}\right)$ & $63 \pm 27$ \\
\hline LVEF $(\%)$ & $36 \pm 9$ \\
\hline Stroke volume $(\mathrm{mL})$ & $64 \pm 18$ \\
\hline Cardiac index $\left(\mathrm{L} / \mathrm{min} / \mathrm{m}^{2}\right)$ & $2.3 \pm 0.5$ \\
\hline TAPSE $(\mathrm{mm})$ & $19 \pm 4$ \\
\hline
\end{tabular}

The comparison between patients with persistently normal diastolic function, reversible DD, worsening, and persistently abnormal diastolic function is shown in Table 2. Kaplan-Meier curves showed that changes in LV diastolic function from baseline to follow-up examination were strongly associated with the clinical outcome (Fig. 2, p < 0.0001). Patients with worsening or persistently abnormal diastolic function had a significantly higher risk of events than those with persistently normal diastolic function or reversible DD ( $p<0.0001$ for all pair-wise comparisons). Pooling patients with worsening or persistently abnormal diastolic function and stratifying them according to normal or abnormal $\mathrm{Cl}$ (Table 3 ) showed the latter to have a worse outcome than those with normal $\mathrm{Cl}$ (Fig. 3). Notably, when patients were categorized into those with no DD at baseline, those with DD at baseline but recovered at follow-up, and those with DD at baseline that persisted at follow-up, the last two groups showed opposite changes in $\mathrm{Cl}$ at follow-up (Fig. 4). 
Table 2

Comparison between patients with persistently normal diastolic function, reversible DD, worsening and persistently abnormal diastolic function

\begin{tabular}{|c|c|c|c|c|c|}
\hline Variable & $\begin{array}{l}\text { Persistently } \\
\text { normal }(n=154)\end{array}$ & $\begin{array}{l}\text { Reversible } \\
(n=55)\end{array}$ & $\begin{array}{l}\text { Worsening } \\
(n=30)\end{array}$ & $\begin{array}{l}\text { Persistently } \\
\text { abnormal ( } \mathrm{n}= \\
\text { 118) }\end{array}$ & $\begin{array}{l}\mathrm{p}- \\
\text { value }\end{array}$ \\
\hline Age (years) & $65 \pm 11$ & $67 \pm 10$ & $73 \pm 10 *$ & $71 \pm 12^{*}$ & $<.0001$ \\
\hline $\begin{array}{l}\text { Systolic blood } \\
\text { pressure }(\mathrm{mmHg})\end{array}$ & $125 \pm 16$ & $126 \pm 18$ & $128 \pm 20$ & $120 \pm 18 *$ & 0.01 \\
\hline $\begin{array}{l}\text { Diastolic blood } \\
\text { pressure (mmHg) }\end{array}$ & $75 \pm 9$ & $75 \pm 10$ & $73 \pm 9$ & $72 \pm 10$ & 0.06 \\
\hline $\begin{array}{l}\text { Mean blood pressure } \\
(\mathrm{mmHg})\end{array}$ & $92 \pm 10$ & $92 \pm 11$ & $91 \pm 10$ & $88 \pm 11^{*}$ & 0.02 \\
\hline Creatinine (mg/dL) & $1.1 \pm 0.4$ & $1.1 \pm 0.3$ & $1.2 \pm 0.6$ & $1.3 \pm 0.6^{*}$ & 0.001 \\
\hline $\begin{array}{l}\text { MDRD } \\
\left(\mathrm{ml} / \mathrm{min} / 1.73 \mathrm{~m}^{2}\right)\end{array}$ & $74 \pm 24$ & $70 \pm 20$ & $68 \pm 30$ & $64 \pm 25^{\star}$ & 0.005 \\
\hline Weight (kg) & $81 \pm 17$ & $77 \pm 17$ & $79 \pm 18$ & $80 \pm 16$ & 0.5 \\
\hline $\operatorname{BSA}\left(\mathrm{m}^{2}\right)$ & $1.9 \pm 0.2$ & $1.9 \pm 0.2$ & $1.9 \pm 0.2$ & $1.9 \pm 0.2$ & 0.5 \\
\hline $\mathrm{BMI}\left(\mathrm{kg} / \mathrm{m}^{2}\right)$ & $28 \pm 5$ & $26 \pm 5$ & $27 \pm 6$ & $27 \pm 5$ & 0.3 \\
\hline \multicolumn{6}{|l|}{$\begin{array}{l}\text { Echocardiography at } \\
\text { baseline }\end{array}$} \\
\hline Heart rate (bpm) & $68 \pm 10$ & $72 \pm 13$ & $70 \pm 10$ & $73 \pm 12^{\star}$ & 0.002 \\
\hline $\mathrm{E}(\mathrm{cm} / \mathrm{s})$ & $61 \pm 21$ & $81 \pm 22^{\star}$ & $67 \pm 16^{\wedge}$ & $93 \pm 25^{\star \wedge} \S$ & $\begin{array}{l}<.0001 \\
0.00\end{array}$ \\
\hline $\mathrm{A}(\mathrm{cm} / \mathrm{s})$ & $86 \pm 21$ & $76 \pm 28$ & $90 \pm 21$ & $61 \pm 29 * \wedge \S$ & $<.0001$ \\
\hline$E / A$ & $0.6 \pm 0.3$ & $1.2 \pm 0.9 *$ & $0.6 \pm 0.4^{\wedge}$ & $1.5 \pm 1.4 * \S$ & $<.0001$ \\
\hline $\begin{array}{l}\text { Deceleration time } \\
\text { (ms) }\end{array}$ & $208 \pm 61$ & $166 \pm 61^{\star}$ & $195 \pm 70$ & $148 \pm 53 * \S$ & $\begin{array}{l}<.0001 \\
0.00\end{array}$ \\
\hline$E / e^{\prime}$ & $10 \pm 3$ & $15 \pm 5^{\star}$ & $12 \pm 4^{\wedge}$ & $18 \pm 7^{\star \wedge} \S$ & $<.0001$ \\
\hline
\end{tabular}

${ }^{*} \mathrm{p}<0.05$ vs persistently normal

${ }^{\wedge} \mathrm{p}<0.05$ vs reversible

$\S p<0.05$ vs worsening 


\begin{tabular}{|c|c|c|c|c|c|}
\hline Variable & $\begin{array}{l}\text { Persistently } \\
\text { normal }(n=154)\end{array}$ & $\begin{array}{l}\text { Reversible } \\
(n=55)\end{array}$ & $\begin{array}{l}\text { Worsening } \\
(n=30)\end{array}$ & $\begin{array}{l}\text { Persistently } \\
\text { abnormal }(n= \\
118)\end{array}$ & $\begin{array}{l}\text { p- } \\
\text { value }\end{array}$ \\
\hline LAVi $\left(\mathrm{mL} / \mathrm{m}^{2}\right)$ & $34 \pm 11$ & $45 \pm 14^{\star}$ & $39 \pm 16$ & $53 \pm 18^{\star \wedge} \S$ & $\begin{array}{l}< \\
0.0001\end{array}$ \\
\hline sPAP (mmHg) & $31 \pm 8$ & $38 \pm 10^{*}$ & $34 \pm 10$ & $46 \pm 11^{\star \wedge} \S$ & $\hat{0} .0001$ \\
\hline EDVi $\left(\mathrm{mL} / \mathrm{m}^{2}\right)$ & $91 \pm 22$ & $105 \pm 29 *$ & $108 \pm 38^{*}$ & $106 \pm 28^{*}$ & $\hat{0} .0001$ \\
\hline ESVi $\left(\mathrm{mL} / \mathrm{m}^{2}\right)$ & $59 \pm 20$ & $73 \pm 24^{\star}$ & $77 \pm 37 *$ & $75 \pm 24^{*}$ & $\begin{array}{l}< \\
0.0001\end{array}$ \\
\hline $\operatorname{LVEF}(\%)$ & $36 \pm 7$ & $31 \pm 6^{*}$ & $31 \pm 9 *$ & $31 \pm 7^{*}$ & $\begin{array}{l}<.0001 \\
0\end{array}$ \\
\hline Stroke volume (mL) & $68 \pm 15$ & $63 \pm 19$ & $67 \pm 18$ & $61 \pm 18^{*}$ & 0.007 \\
\hline $\begin{array}{l}\text { Cardiac index } \\
\left(\mathrm{L} / \mathrm{min} / \mathrm{m}^{2}\right)\end{array}$ & $2.3 \pm 0.4$ & $2.4 \pm 0.5$ & $2.4 \pm 0.6$ & $2.3 \pm 0.5$ & 0.2 \\
\hline TAPSE (mm) & $20 \pm 4$ & $19 \pm 3$ & $20 \pm 4$ & $17 \pm 3^{\star \wedge} \S$ & $\begin{array}{l}<.0001 \\
0\end{array}$ \\
\hline \multicolumn{6}{|l|}{$\begin{array}{l}\text { Echocardiography at } \\
\text { follow-up }\end{array}$} \\
\hline Heart rate (bpm) & $68 \pm 11$ & $71 \pm 11$ & $73 \pm 10$ & $77 \pm 14^{\star \wedge}$ & $\hat{0} .0001$ \\
\hline $\mathrm{E}(\mathrm{cm} / \mathrm{s})$ & $62 \pm 18$ & $65 \pm 16$ & $87 \pm 23^{\star \wedge}$ & $97 \pm 26^{\star \wedge}$ & $\hat{0} .0001$ \\
\hline $\mathrm{A}(\mathrm{cm} / \mathrm{s})$ & $83 \pm 22$ & $86 \pm 26$ & $75 \pm 24$ & $60 \pm 31^{\star \wedge} \S$ & $<.0001$ \\
\hline $\mathrm{E} / \mathrm{A}$ & $0.8 \pm 0.3$ & $0.8 \pm 0.4$ & $1.3 \pm 0.8^{*}$ & $2.1 \pm 1.4^{\star \wedge} \S$ & $\hat{0} .0001$ \\
\hline $\begin{array}{l}\text { Deceleration time } \\
\text { (ms) }\end{array}$ & $210 \pm 62$ & $199 \pm 70$ & $167 \pm 62^{\star}$ & $147 \pm 55^{\star \wedge}$ & $\begin{array}{l}<.0001 \\
0.01\end{array}$ \\
\hline$E / e^{\prime}$ & $10 \pm 4$ & $11 \pm 3$ & $17 \pm 5^{\star \wedge}$ & $19 \pm 6^{\star 1}$ & $\begin{array}{l}<.0001 \\
0.00\end{array}$ \\
\hline $\mathrm{LAVi}\left(\mathrm{mL} / \mathrm{m}^{2}\right)$ & $34 \pm 13$ & $37 \pm 12$ & $50 \pm 16^{\star \wedge}$ & $58 \pm 18^{* \wedge}$ & $\begin{array}{l}<.0001 \\
0.01\end{array}$ \\
\hline \multicolumn{6}{|c|}{${ }^{*} \mathrm{p}<0.05$ vs persistently normal } \\
\hline \multicolumn{6}{|l|}{${ }^{\wedge} p<0.05$ vs reversible } \\
\hline$\S p<0.05$ vs worsenin & & & & & \\
\hline
\end{tabular}




\begin{tabular}{|c|c|c|c|c|c|}
\hline Variable & $\begin{array}{l}\text { Persistently } \\
\text { normal }(n=154)\end{array}$ & $\begin{array}{l}\text { Reversible } \\
(n=55)\end{array}$ & $\begin{array}{l}\text { Worsening } \\
(n=30)\end{array}$ & $\begin{array}{l}\text { Persistently } \\
\text { abnormal ( } \mathrm{n}= \\
118)\end{array}$ & $\begin{array}{l}\mathrm{p}- \\
\text { value }\end{array}$ \\
\hline $\mathrm{sPAP}(\mathrm{mmHg})$ & $30 \pm 5$ & $33 \pm 6$ & $40 \pm 9 * \wedge$ & $44 \pm 12^{\star \wedge}$ & $<.0001$ \\
\hline $\operatorname{EDVi}\left(\mathrm{mL} / \mathrm{m}^{2}\right)$ & $85 \pm 22$ & $85 \pm 19$ & $112 \pm 42^{\star \wedge}$ & $107 \pm 29 * \wedge$ & <. 0001 \\
\hline ESVi $\left(\mathrm{mL} / \mathrm{m}^{2}\right)$ & $53 \pm 20$ & $54 \pm 17$ & $80 \pm 39 * \wedge$ & $76 \pm 27^{\star \wedge}$ & $\begin{array}{l}<.0001 \\
0.0\end{array}$ \\
\hline LVEF (\%) & $39 \pm 8$ & $38 \pm 7$ & $31 \pm 8^{\star \wedge}$ & $30 \pm 8^{\star \wedge}$ & $\begin{array}{l}< \\
0.0001\end{array}$ \\
\hline Stroke volume $(\mathrm{mL})$ & $69 \pm 14$ & $69 \pm 17$ & $62 \pm 19$ & $57 \pm 19 * \wedge$ & $\begin{array}{l}< \\
0.0001\end{array}$ \\
\hline $\begin{array}{l}\text { Cardiac index } \\
\left(\mathrm{L} / \mathrm{min} / \mathrm{m}^{2}\right)\end{array}$ & $2.4 \pm 0.4$ & $2.5 \pm 0.5$ & $2.3 \pm 0.5$ & $2.1 \pm 0.5^{\star \wedge}$ & $\begin{array}{l}< \\
0.0001\end{array}$ \\
\hline TAPSE (mm) & $21 \pm 4$ & $20 \pm 3$ & $19 \pm 4$ & $17 \pm 4^{\star \wedge}$ & $\begin{array}{l}< \\
0.0001\end{array}$ \\
\hline \multicolumn{6}{|c|}{${ }^{*} \mathrm{p}<0.05$ vs persistently normal } \\
\hline \multicolumn{6}{|l|}{${ }^{\wedge} p<0.05$ vs reversible } \\
\hline$\S p<0.05$ vs worsenir & & & & & \\
\hline
\end{tabular}


Table 3

Group 1 (patients with either persistently abnormal DD or worsening diastolic function and normal $\mathrm{Cl}$ at follow-up) vs Group 2 (patients with either persistently abnormal DD or worsening diastolic function and reduced $\mathrm{Cl}$ at follow-up)

\begin{tabular}{|c|c|c|c|}
\hline Variable & $\begin{array}{l}\text { Group } 1 \\
(n=96)\end{array}$ & $\begin{array}{l}\text { Group } 2 \\
(n=52)\end{array}$ & p-value \\
\hline Age (years) & $72 \pm 11$ & $71 \pm 13$ & 0.8 \\
\hline Systolic blood pressure (mmHg) & $124 \pm 18$ & $115 \pm 17$ & 0.003 \\
\hline Diastolic blood pressure (mmHg) & $73 \pm 9$ & $71 \pm 10$ & 0.3 \\
\hline Mean blood pressure (mmHg) & $90 \pm 11$ & $86 \pm 11$ & 0.02 \\
\hline Creatinine (mg/dL) & $1.3 \pm 0.5$ & $1.4 \pm 0.7$ & 0.3 \\
\hline MDRD (ml/min/1.73m²) & $66 \pm 26$ & $62 \pm 25$ & 0.4 \\
\hline Weight (kg) & $79 \pm 16$ & $82 \pm 17$ & 0.3 \\
\hline $\operatorname{BSA}\left(\mathrm{m}^{2}\right)$ & $1.9 \pm 0.2$ & $1.9 \pm 0.2$ & 0.3 \\
\hline BMI $\left(\mathrm{kg} / \mathrm{m}^{2}\right)$ & $27 \pm 5$ & $28 \pm 5$ & 0.3 \\
\hline \multicolumn{4}{|l|}{ Echocardiography at baseline } \\
\hline Heart rate (bpm) & $73 \pm 11$ & $73 \pm 13$ & 0.9 \\
\hline $\mathrm{E}(\mathrm{cm} / \mathrm{s})$ & $90 \pm 27$ & $84 \pm 23$ & 0.2 \\
\hline $\mathrm{A}(\mathrm{cm} / \mathrm{s})$ & $74 \pm 30$ & $57 \pm 27$ & 0.005 \\
\hline$E / A$ & $1.1 \pm 1.1$ & $1.3 \pm 1.1$ & 0.6 \\
\hline Deceleration time (ms) & $160 \pm 62$ & $152 \pm 56$ & 0.4 \\
\hline$E / e^{\prime}$ & $17 \pm 7$ & $17 \pm 6$ & 0.6 \\
\hline LAVi $\left(\mathrm{mL} / \mathrm{m}^{2}\right)$ & $50 \pm 19$ & $50 \pm 18$ & 0.9 \\
\hline sPAP (mmHg) & $42 \pm 11$ & $46 \pm 12$ & 0.06 \\
\hline EDVi $\left(m L / m^{2}\right)$ & $106 \pm 30$ & $109 \pm 31$ & 0.6 \\
\hline ESVi $\left(\mathrm{mL} / \mathrm{m}^{2}\right)$ & $73 \pm 27$ & $80 \pm 28$ & 0.1 \\
\hline LVEF (\%) & $32 \pm 7$ & $28 \pm 7$ & 0.001 \\
\hline Stroke volume $(\mathrm{mL})$ & $68 \pm 17$ & $52 \pm 15$ & $<0.0001$ \\
\hline
\end{tabular}




\begin{tabular}{|c|c|c|c|}
\hline Variable & $\begin{array}{l}\text { Group } 1 \\
(n=96)\end{array}$ & $\begin{array}{l}\text { Group } 2 \\
(n=52)\end{array}$ & p-value \\
\hline Cardiac index $\left(\mathrm{L} / \mathrm{min} / \mathrm{m}^{2}\right)$ & $2.5 \pm 0.5$ & $1.9 \pm 0.4$ & $<0.0001$ \\
\hline TAPSE (mm) & $18 \pm 3$ & $17 \pm 4$ & 0.02 \\
\hline \multicolumn{4}{|c|}{ Echocardiography at follow-up } \\
\hline Heart rate (bpm) & $74 \pm 13$ & $80 \pm 15$ & 0.01 \\
\hline$E(\mathrm{~cm} / \mathrm{s})$ & $96 \pm 27$ & $94 \pm 22$ & 0.5 \\
\hline $\mathrm{A}(\mathrm{cm} / \mathrm{s})$ & $71 \pm 31$ & $48 \pm 21$ & 0.1 \\
\hline $\mathrm{E} / \mathrm{A}$ & $1.7 \pm 1.4$ & $2.3 \pm 1.1$ & 0.06 \\
\hline Deceleration time (ms) & $159 \pm 61$ & $138 \pm 47$ & 0.03 \\
\hline$E / e^{\prime}$ & $18 \pm 6$ & $20 \pm 5$ & 0.03 \\
\hline LAVi $\left(\mathrm{mL} / \mathrm{m}^{2}\right)$ & $56 \pm 17$ & $56 \pm 19$ & 0.8 \\
\hline sPAP $(\mathrm{mmHg})$ & $41 \pm 10$ & $45 \pm 13$ & 0.04 \\
\hline $\operatorname{EDVi}\left(\mathrm{mL} / \mathrm{m}^{2}\right)$ & $106 \pm 30$ & $113 \pm 34$ & 0.2 \\
\hline ESVi $\left(\mathrm{mL} / \mathrm{m}^{2}\right)$ & $73 \pm 28$ & $85 \pm 32$ & 0.02 \\
\hline LVEF (\%) & $33 \pm 7$ & $27 \pm 8$ & $<0.0001$ \\
\hline Stroke volume (mL) & $66 \pm 18$ & $43 \pm 11$ & $<0.0001$ \\
\hline Cardiac index $\left(\mathrm{L} / \mathrm{min} / \mathrm{m}^{2}\right)$ & $2.5 \pm 0.4$ & $1.7 \pm 0.2$ & $<0.0001$ \\
\hline TAPSE (mm) & $19 \pm 4$ & $16 \pm 4$ & $<0.0001$ \\
\hline$\# p<0.05$ vs Group 1 & & & \\
\hline
\end{tabular}

ROC analysis across nested models also showed a progressive increase in the area under the curve considering an initial model including only clinical variables, then adding natriuretic peptides, echocardiographic variables and changes in LV diastolic function (Fig. 5). Cox regression analysis also showed that LV diastolic function changes were associated with the clinical endpoint, independent of confounders (Supplemental Table 1). The incremental analysis confirmed that LV diastolic function changes added significant prognostic information to a comprehensive model, including clinical variables, natriuretic peptide, and echocardiographic variables (Supplemental Fig. 5).

\section{Discussion}


Repeated assessment of LV diastolic function and $\mathrm{Cl}$ helps in risk stratification of chronic HF outpatients. Patients with recovered DD at follow-up had a significantly better clinical outcome, whereas those with either persistently abnormal or worsening diastolic function most often had an adverse outcome. Prognosis worsened in patients exhibiting both DD and compromised $\mathrm{Cl}$ at follow-up.

\section{The prognostic value of left ventricular filling pressure and its change over time}

Much has been written about the value of reducing LVFP to improve patient's clinical outcome, with Doppler echocardiographic markers showing normalized LVFP at rest and with stress associated with better survival[15, 16]; however, some controversies remain. Recent data showing lower rates of HF and all-cause hospitalizations during the year after implantation of the CardioMEMS pulmonary artery pressure sensor boosted the role of repeated assessment of LVFP to guide optimum therapy for ambulatory HF patients[17].

Echocardiography has become the most important investigation for HF patients since it provides LV function measures, both systolic and diastolic. Several parameters related to hemodynamic variables, including LVFP, have been recognized and reported. A restrictive LV filling pattern (i.e., a short isovolumic relaxation time, mitral EDT $<150 \mathrm{~ms}$ ) and an averaged E/e' $\geq 13$ reliably identify elevated filling pressure, particularly in patients with compromised LV EF $[18,19]$. Current HF guidelines do not recommend periodically repeated echocardiograms in the follow-up of clinically stable patients, with reassessing myocardial structure and functions is only warranted when patients experience worsening episodes[20]. However, some available studies have shown that repeated evaluations with Doppler echocardiography can be of prognostic relevance in HF patients. The estimation of LVFP by echocardiography may be accomplished using the 2016 ASE/EACVI recommendations [21]. Still, to the best of our knowledge, no study addressed the predictive value of either elevated LVFP or reversing DD during follow-up in ambulatory HF patients evaluated by the algorithm proposed by the guidelines and submitted to optimized drug therapy with diuretics and evidence-based medications.

In our study, the presence of echocardiographic evidence for DD at follow-up, resulting from persistently abnormal or worsening diastolic function, provided incremental prognostic information over and above that given by a comprehensive evaluation of clinical and demographic variables, absolute or relative NP target levels, and other echocardiographic parameters. Interestingly, persistently normal diastolic function and the reversal of DD with therapy were associated with a better outcome.

\section{The role of compromised forward flow and of its changes over time}

Low-output HF represents a hallmark of advanced HF that is not infrequent in patients with chronic $\mathrm{HF}[22]$; however, scanty information is available on the prognostic value of non-invasively measured $\mathrm{CO}$ and related parameters. Since Doppler echocardiography allows for quantitative assessment of forward 
flow, a comprehensive echocardiographic examination incorporating measurement of stroke volume, $\mathrm{CO}$, and $\mathrm{Cl}$ should be easily obtained $[23,24]$.

According to the available literature, a classification guided by LVFP and $\mathrm{Cl}$ baseline measures can be obtained to estimate the patient's hemodynamic status and advise on treatment optimization[25, 26]. Therefore, echo-directed categorization of chronic HF patients according to hemodynamic profiles, i.e., no $\mathrm{DD}, \mathrm{DD}$ with normal $\mathrm{Cl}$ and $\mathrm{DD}$ with reduced $\mathrm{Cl}$, can provide detailed information on both baseline conditions and changes that occur over time. In this study, we addressed the prognostic importance of the changes of systolic and diastolic parameters during ambulatory patients' follow-up. Patients with either persistently abnormal or worsening diastolic function and reduced $\mathrm{Cl}$ had the worst prognosis. In contrast, those whose DD recovered during the follow-up demonstrated amelioration of forward flow that likely contributed to the improved clinical status and prognosis. The finding of the increase in $\mathrm{CO}$ in patients showing diastolic function recovery, as a result of tailored therapy, is not new but consistent with previous observations that reported an association of improved diastolic function with higher $\mathrm{CO}$ in patients with HF, irrespective of LV EF. Nevertheless, our finding was the first to describe it using echocardiography in chronic HF and the ambulatory setting. Several mechanisms may be invoked to explain the latter result, including an actual increase in myocardial contractility, a decrease in functional mitral regurgitation with a redistribution of intracardiac volumes to supplement the forward flow, or the heart's ability to increase the forward flow in response to a decrease in LVFP, as most dilated ventricles operate far beyond the level of LVFP at which stroke volume depends on increments of LVFP.

\section{Clinical implications}

A comprehensive echocardiographic assessment of LVFP and forward flow has recently shown the potential of characterizing the hemodynamic status and prognosis of patients with acute HF. With advances in outpatient management for stable ambulatory patients, new challenges are now seen, most notably preventing clinical decompensation and cardiac-related death[27-31]. It has been demonstrated that the recovery of LVFP during clinical follow-up can improve morbidity and mortality in patients with $\mathrm{HF}[32]$. The present study was an attempt to demonstrate the value of repeated echocardiographic evaluation to obtain a simultaneous picture of the patient's hemodynamic profile and eventually to guide optimum therapy. In contrast with a previous report that comprised only a limited number of echo variables[33-35], our study results showed that a comprehensive assessment of repeatedly measured echocardiographic hemodynamic parameters, such as LVFP and $\mathrm{Cl}$, may help risk stratification in patients with chronic HF.

\section{Limitations}

The optimal timing of repeated echocardiography remains uncertain, probably depending on individual patient's clinical condition. Although novel classifications of HF by echocardiography have been proposed[36], in the present study, we used a $\mathrm{Cl}$ of $2.0 \mathrm{l} / \mathrm{min} / \mathrm{m}^{2}$ as a cutoff of forward flow based on a hemodynamic study indicating that peripheral hypoperfusion at $\mathrm{Cl}$ level at or below $2.0 \mathrm{l} / \mathrm{min} / \mathrm{m}^{2}$ was associated with an adverse outcome[9]. Estimating stroke volume is prone to measurement errors, that 
can be amplified extrapolating measures such as LVOT velocity-time integral into hemodynamic variables such as $\mathrm{Cl}$. However, the echocardiographic data reported and analyzed in this study were collected in accordance with contemporary quality standards and reflect the data measured in clinical practice which are regularly utilized for decision-making such as calculations of aortic valve area in patients with aortic stenosis. Other studies are warranted to assess hemodynamic profiles that incorporate right ventricular function and strain imaging measures in patients with chronic HF.

\section{Conclusions}

The use of serial echocardiography evaluation helps in risk stratification of HFrEF patients. This study seems to favour the systematic use of a 2-point observation system separated by 3 to 6 months for assessing prognosis.

\section{References}

1. Mele D (2019) From left ventricular ejection fraction to cardiac hemodynamics: role of echocardiography in evaluating patients with heart failure

2. Lester SJ, Tajik AJ, Nishimura RA, et al (2008) Unlocking the Mysteries of Diastolic Function Deciphering the Rosetta Stone 10 Years Later. 51:. https://doi.org/10.1016/j.jacc.2007.09.061

3. Pozzoli M, Capomolla S, Pinna G, et al (1996) Doppler echocardiography reliably predicts pulmonary artery wedge pressure in patients with chronic heart failure with and without mitral regurgitation. $J$ Am Coll Cardiol 27:883-893. https://doi.org/10.1016/0735-1097(95)00553-6

4. Pinamonti B, Zecchin M, Lenarda ADI, et al (1997) Persistence of Restrictive Left Ventricular Filling Pattern in Dilated Cardiomyopathy: An Ominous Prognostic Sign. J Am Coll Cardiol 29:604-612. https://doi.org/10.1016/S0735-1097(96)00539-6

5. Whalley GA, Mhs C, Doughty RN, et al (2002) Pseudonormal Mitral Filling Pattern Predicts Hospital Re-Admission in Patients With Congestive Heart Failure. J Am Coll Cardiol 39:1787-1795. https://doi.org/10.1016/S0735-1097(02)01868-5

6. Lancellotti P, Galderisi M, Edvardsen T, et al (2017) Echo-Doppler estimation of left ventricular filling pressure: results of the multicentre EACVI Euro-Filling study. Eur Hear $\mathrm{J}$ - Cardiovasc Imaging 1-8. https://doi.org/10.1093/ehjci/jex067

7. Tobushi T, Nakano M, Hosokawa K, et al (2017) Improved Diastolic Function Is Associated With Higher Cardiac Output in Patients With Heart Failure Irrespective of Left Ventricular Ejection Fraction. 1-7. https://doi.org/10.1161/JAHA.116.003389

8. Stevenson LW, Tillisch JH (1986) Maintenance of cardiac output with normal filling pressures in patients with dilated heart failure. Circulation 74:1303-1308.

https://doi.org/10.1161/01.CIR.74.6.1303

9. Cooper LB, Mentz RJ, Stevens SR, et al (2016) Hemodynamic Predictors of Heart Failure Morbidity and Mortality: Fluid or Flow? J Card Fail 22:182-189. https://doi.org/10.1016/j.cardfail.2015.11.012 
10. Hamdan R, Charif F, Zein A, Issa M, Najjar C, Abdallah H, Fakih S SM (2019) Noninvasive Monitoring of Cardiac Output: A Useful Tool Yet? J Cardiovasc Echogr Oct-Dec;29:

11. Nagueh SF, Smiseth OA, Appleton CP, et al (2016) Recommendations for the Evaluation of Left Ventricular Diastolic Function by Echocardiography: An Update from the American Society of Echocardiography and the European Association of Cardiovascular Imaging. J Am Soc Echocardiogr 29:277-314. https://doi.org/10.1016/j.echo.2016.01.011

12. Hahn RT, Pibarot P (2017) Accurate Measurement of Left Ventricular Outflow Tract Diameter: Comment on the Updated Recommendations for the Echocardiographic Assessment of Aortic Valve Stenosis. J Am Soc Echocardiogr 30:1038-1041. https://doi.org/10.1016/j.echo.2017.06.002

13. Barbier P, Cucco C, Guglielmo M, et al (2020) Estimation of increased pulmonary wedge pressure by an algorithm based on noninvasively measured pulmonary diastolic pressure in cardiac patients independent of left ventricular ejection fraction. Echocardiography 37:215-222. https://doi.org/10.1111/echo.14581

14. Ibrahim NE, Burnett JC, Butler J, et al (2020) Natriuretic Peptides as Inclusion Criteria in Clinical Trials: A JACC: Heart Failure Position Paper. JACC Hear Fail 8:347-358. https://doi.org/10.1016/j.jchf.2019.12.010

15. Stevenson LW (1999) Tailored therapy to hemodynamic goals for advanced heart failure. Eur J Heart Fail 1:251-257. https://doi.org/10.1016/S1388-9842(99)00015-X

16. Duncan AM, Lim E, Gibson DG, Henein MY (2005) Effect of dobutamine stress on left ventricular filling in ischemic dilated cardiomyopathy: Pathophysiology and prognostic implications. J Am Coll Cardiol 46:488-496. https://doi.org/10.1016/j.jacc.2005.04.048

17. Heywood JT, Jermyn R, Shavelle D, et al (2017) Impact of Practice-Based Management of Pulmonary Artery Pressures in 2000 Patients Implanted with the CardioMEMS Sensor. Circulation 135:15091517. https://doi.org/10.1161/CIRCULATIONAHA.116.026184

18. Temporelli PL, Scapellato F, Eleuteri E, et al (2010) Doppler echocardiography in advanced systolic heart failure: A noninvasive alternative to Swan-Ganz Catheter. Circ Hear Fail 3:387-394. https://doi.org/10.1161/CIRCHEARTFAILURE.108.809590

19. Nagueh SF, Bhatt R, Vivo RP, et al (2011) Echocardiographic evaluation of hemodynamics in patients with decompensated systolic heart failure. Circ Cardiovasc Imaging 4:220-227. https://doi.org/10.1161/CIRCIMAGING.111.963496

20. Yancy CW, Jessup M, Bozkurt B, et al (2017) 2017 ACC/AHA/HFSA Focused Update of the 2013 ACCF/AHA Guideline for the Management of Heart Failure: A Report of the American College of Cardiology/American Heart Association Task Force on Clinical Practice Guidelines and the Heart Failure Society of Amer. Circulation 136:e137-e161. https://doi.org/10.1161/CIR.0000000000000509

21. Balaney B, Medvedofsky D, Mediratta A, et al (2018) HHS Public Access. 31:79-88. https://doi.org/10.1016/j.echo.2017.09.002.Invasive 
22. Albakri A (2019) Low-output heart failure: A review of clinical status and meta-analysis of diagnosis and clinical management methods. Clin Med Investig 4:. https://doi.org/10.15761/cmi.1000179

23. Mele D, Pestelli G, Dini FL, et al (2020) Novel Echocardiographic Approach to Hemodynamic Phenotypes Predicts Outcome of Patients Hospitalized with Heart Failure. Circ Cardiovasc Imaging 1-13. https://doi.org/10.1161/CIRCIMAGING.119.009939

24. Abbas AE, Khoury Abdulla R, Aggrawal A, et al (2017) A novel echocardiographic hemodynamic classification of heart failure based on stroke volume index and left atrial pressure. Echocardiography 34:1417-1425. https://doi.org/10.1111/echo.13642

25. Nohria A, Tsang SW, Fang JC, et al (2003) Clinical assessment identifies hemodynamic profiles that predict outcomes in patients admitted with heart failure. J Am Coll Cardiol 41:1797-1804. https://doi.org/10.1016/S0735-1097(03)00309-7

26. Drazner MH, Hellkamp AS, Leier C V., et al (2008) Value of clinician assessment of hemodynamics in advanced heart failure: the ESCAPE trial. Circ Heart Fail 1:170-177. https://doi.org/10.1161/CIRCHEARTFAILURE.108.769778

27. Simioniuc A, Carluccio E, Ghio S, et al (2016) Echo and natriuretic peptide guided therapy improves outcome and reduces worsening renal function in systolic heart failure: An observational study of 1137 outpatients. Int J Cardiol 224:416-423. https://doi.org/10.1016/j.ijcard.2016.09.034

28. Pugliese NR, Fabiani I, Santini C, et al (2019) Value of combined cardiopulmonary and echocardiography stress test to characterize the haemodynamic and metabolic responses of patients with heart failure and mid-range ejection fraction. Eur Heart J Cardiovasc Imaging 20:828836. https://doi.org/10.1093/ehjci/jez014

29. Pugliese NR, Fabiani I, Mandoli GE, et al (2019) Echo-derived peak cardiac power output-to-left ventricular mass with cardiopulmonary exercise testing predicts outcome in patients with heart failure and depressed systolic function. Eur Heart J Cardiovasc Imaging 20:700-708. https://doi.org/10.1093/ehjci/jey172

30. Fabiani I, Pugliese NR, Galeotti GG, et al (2019) The Added Value of Exercise Stress Echocardiography in Patients With Heart Failure. Am J Cardiol 123:1470-1477. https://doi.org/10.1016/j.amjcard.2019.02.008

31. Pugliese NR, De Biase N, Gargani L, et al (2020) Predicting the transition to and progression of heart failure with preserved ejection fraction: a weighted risk score using bio-humoural, cardiopulmonary, and echocardiographic stress testing. Eur J Prev Cardiol. https://doi.org/10.1093/eurjpc/zwaa129

32. Lupón J, Díez-López C, de Antonio M, et al (2017) Recovered heart failure with reduced ejection fraction and outcomes: a prospective study. Eur J Heart Fail 19:1615-1623. https://doi.org/10.1002/ejhf.824

33. van den Berg VJ, Strachinaru M, Akkerhuis KM, et al (2019) Repeated Echocardiograms Do Not Provide Incremental Prognostic Value to Single Echocardiographic Assessment in Minimally Symptomatic Patients with Chronic Heart Failure: Results of the Bio-SHiFT Study. J Am Soc Echocardiogr 32:1000-1009. https://doi.org/10.1016/j.echo.2019.04.419 
34. Tschöpe C, Kasner M (2014) Can speckle-tracking imaging improve the reliability of echocardiographic parameters for outcome evaluation in clinical trials? Eur Heart J 35:605-607. https://doi.org/10.1093/eurheartj/eht217

35. Ritzema JL, Richards AM, Crozier IG, et al (2011) Serial doppler echocardiography and tissue doppler imaging in the detection of elevated directly measured left atrial pressure in ambulant subjects with chronic heart failure. JACC Cardiovasc Imaging 4:927-934.

https://doi.org/10.1016/j.jcmg.2011.07.004

36. Mele D, Andrade A, Bettencourt P, et al (2020) From left ventricular ejection fraction to cardiac hemodynamics: role of echocardiography in evaluating patients with heart failure. Heart Fail. Rev. 25:217-230

\section{Figures}

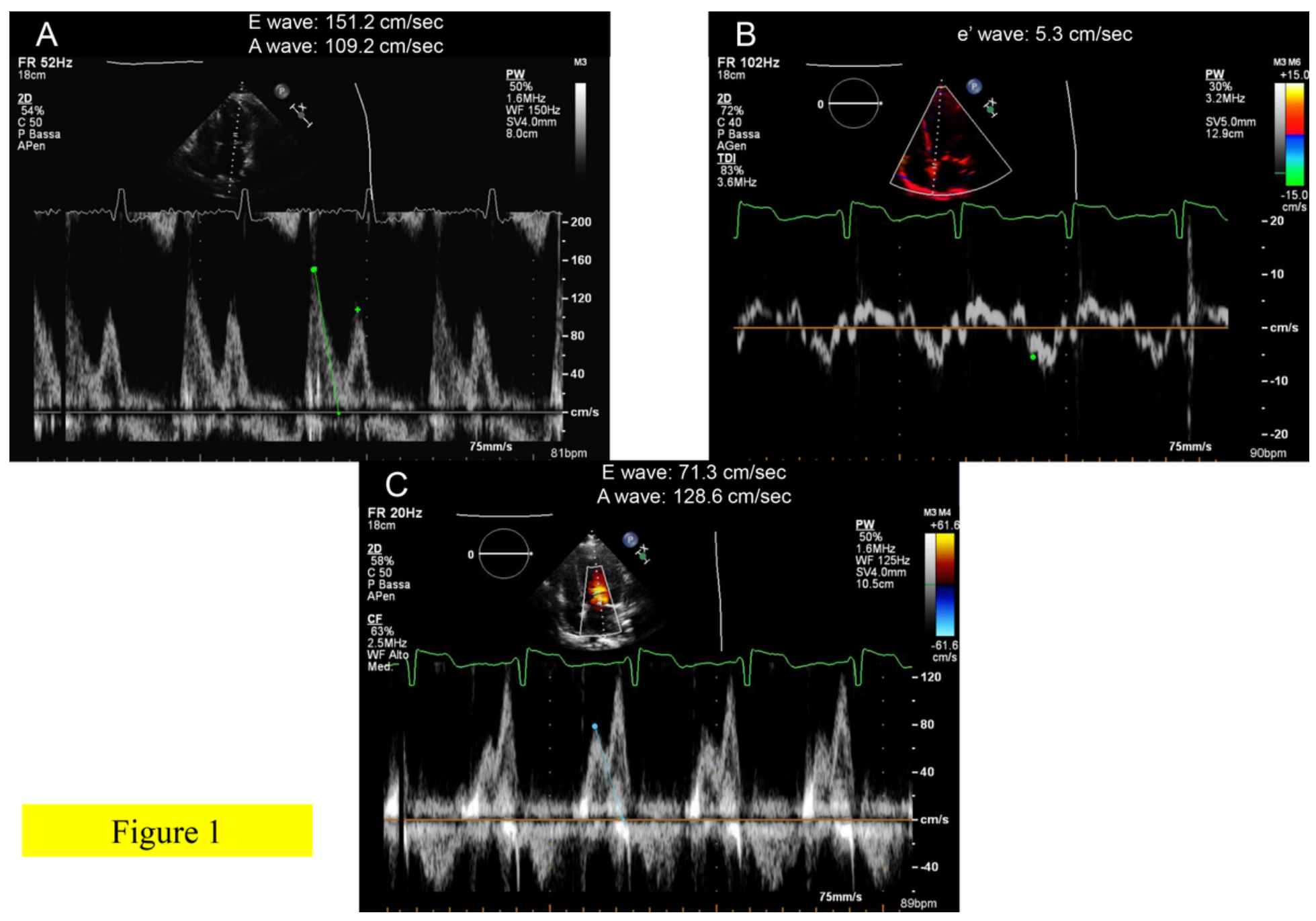

\section{Figure 1}

Baseline spectral Doppler mitral flow recordings of the left ventricular filling (A) and tissue Doppler imaging (B) of mitral annulus indicative of elevated left ventricular filling pressure (averaged E/e' 28.6). 
Reversal of mitral flow at follow-up (C) after optimized tailored therapy (as tissue Doppler imaging was unchanged, E/e' had diminished to 13.4).

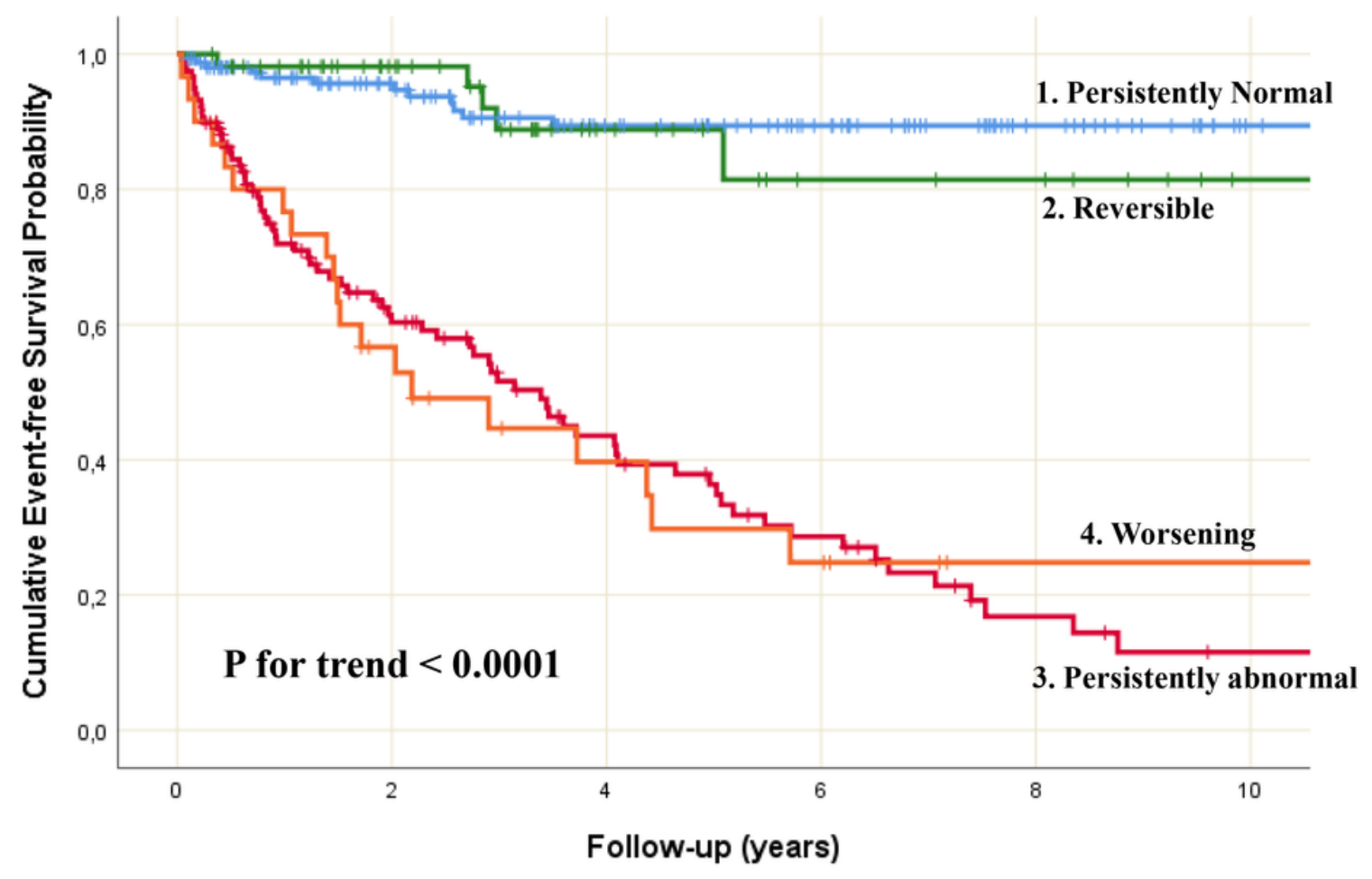

\section{Figure 2}

\section{Figure 2}

Patients with 1) persistently normal diastolic function or 2) reversible diastolic dysfunction had significantly better outcome than 3 ) those with persistenly abnormal diastolic function or 4) worsening diastolic function. Log-rank pair-wise (Mantel-Cox): $p<0.001$ for curve 3 vs 1 or 2; $p<0.001$ for curve 4 vs 1 or $2, p=0.76$ for curve 1 vs $2 ; p=0.78$ for curve 3 vs 4 . 


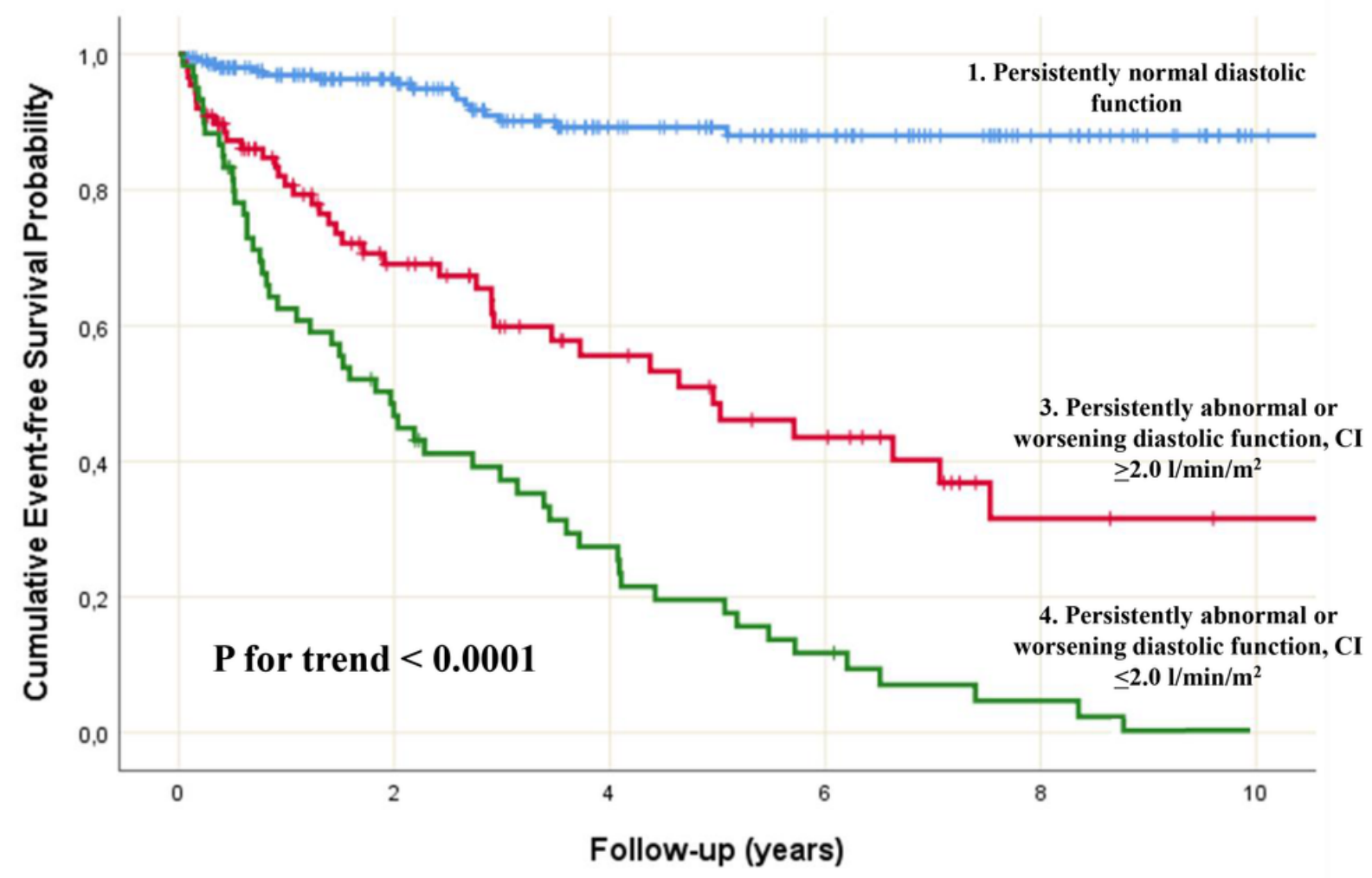

Figure 3

\section{Figure 3}

Survival of patients grouped according to 1) persistently normal diastolic function or reversible diastolic dysfunction, 2) persistently abnormal or worsening diastolic function with cardiac index $\geq 2.0 \mathrm{I} / \mathrm{min} / \mathrm{m} 2$, 3) ) persistently abnormal or worsening diastolic function with cardiac index $<2.0 \mathrm{I} / \mathrm{min} / \mathrm{m} 2$. Abbreviations: Cl: cardiac index. Log-rank pair-wise (Mantel-Cox): $p<0.001$ for all comparisons. 


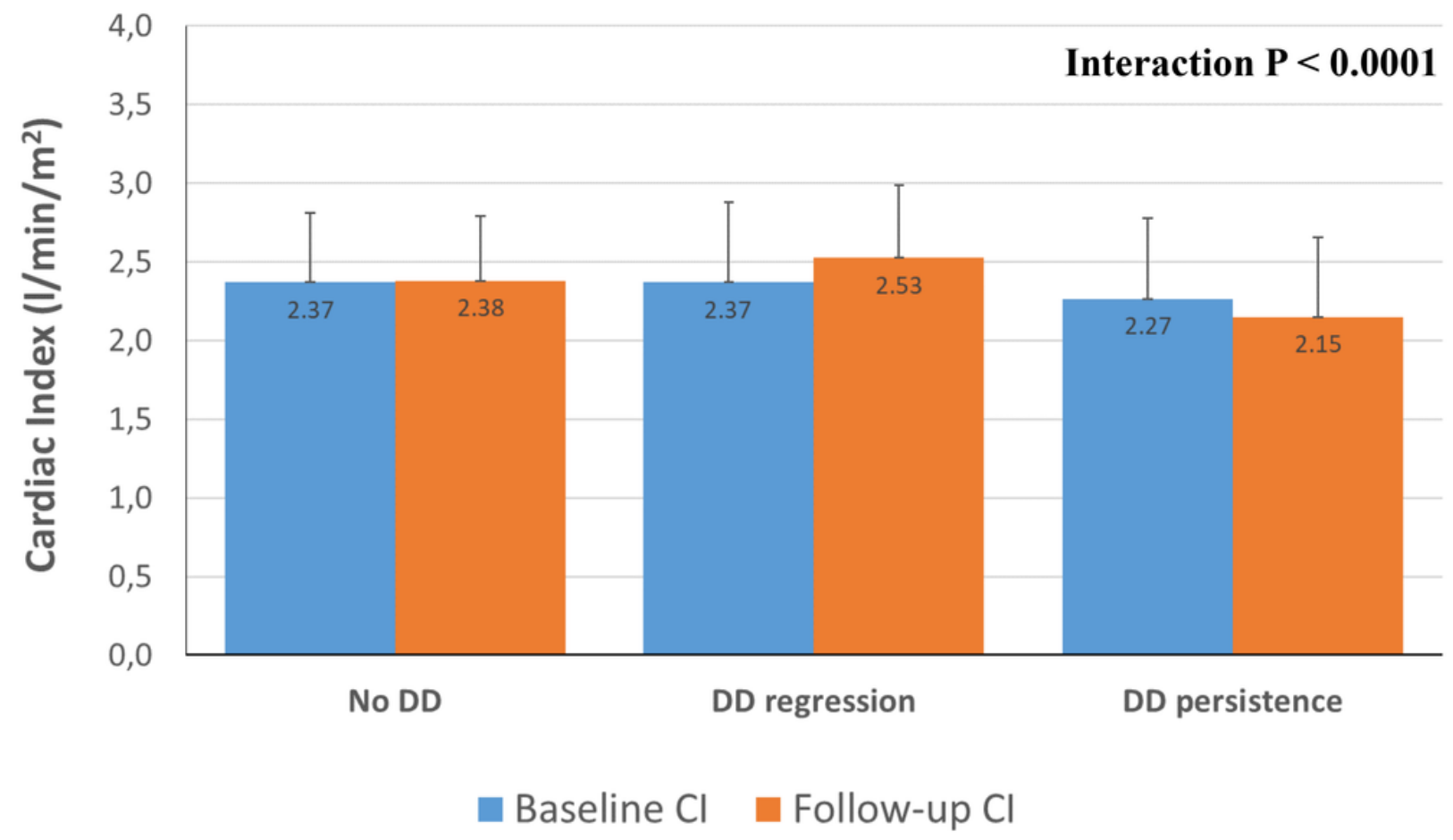

Figure 4

\section{Figure 4}

Effect on cardiac index of regression of diastolic dysfunction and of persistently abnormal or worsening diastolic function compared to patients with persistently normal diastolic function. Abbreviations: $\mathrm{Cl}$ : cardiac index; DD: diastolic dysfunction. 


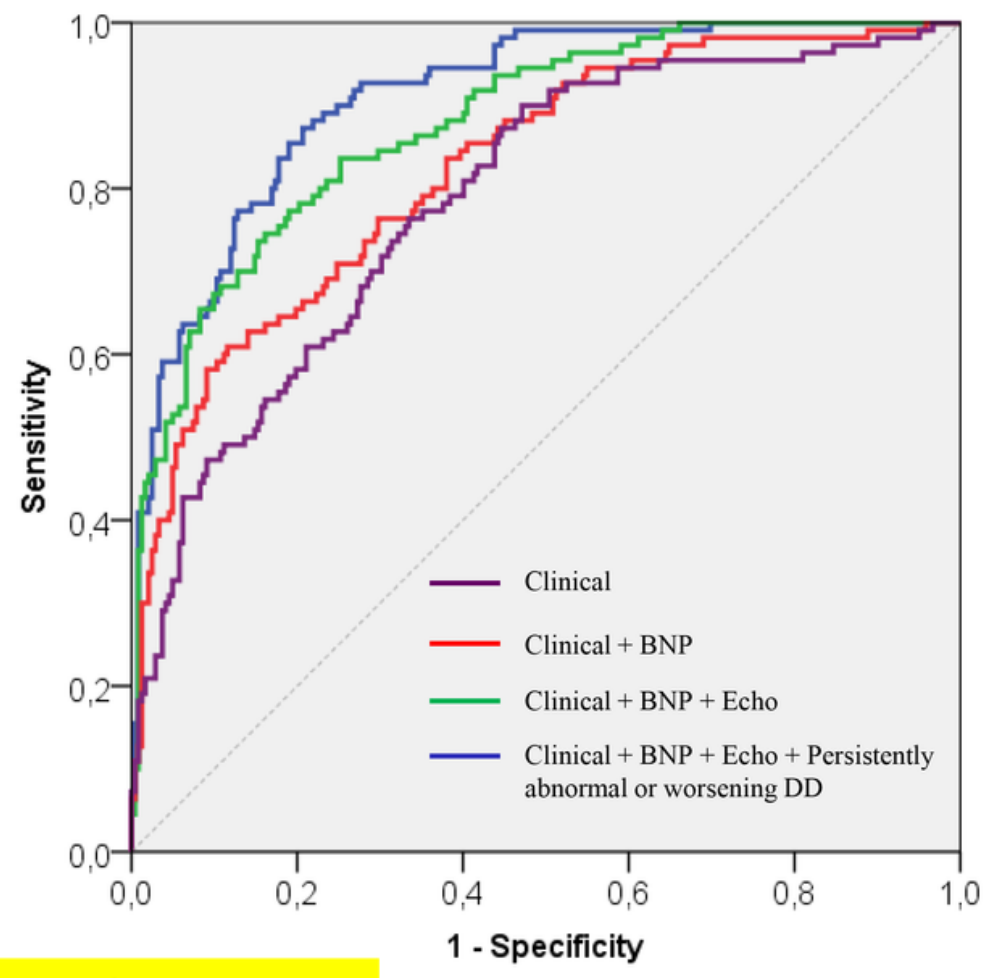

Clinical

AUC 0.79 (0.74-0.84)

Clinical+ BNP

AUC 0.83 (0.78-0.87)

Clinical + Echo

AUC 0.88 (0.84-0.92)

Clinical + Echo +

Persistently abnormal or worsening DD

AUC 0.91 (0.88-0.94)

Figure 5

Figure 5

Discrimination analysis: Area under the curve of the initial model, including clinical and demographic variables, and the contribution of natriuretic peptides, echocardiographic variables and changes in left ventricular diastolic function at the Receiver Operating Characteristic analysis across nested models.

\section{Supplementary Files}

This is a list of supplementary files associated with this preprint. Click to download.

- Supplementalfile.doc

- sup1.png

- sup2.png

- sup3.png

- sup4.png

- sup5.png 